\title{
Stellar Yields of Rotating First Stars: Yields of Weak Supernovae and Abundances of Carbon-enhanced Hyper Metal Poor Stars
}

\author{
Koh Takahashi ${ }^{1}$, Hideyuki Umeda ${ }^{1}$ and Takashi Yoshida ${ }^{2}$ \\ ${ }^{1}$ Department of Astronomy, The University of Tokyo \\ email: ktakahashi@astron.s.u-tokyo.ac.jp \\ ${ }^{2}$ Yukawa Institute for Theoretical Physics, Kyoto University
}

\begin{abstract}
The three most iron-poor stars known until now are also known to have peculiar enhancements of intermediate mass elements. Under the assumption that these iron-deficient stars reveal the nucleosynthesis result of Pop III stars, we show that a weak supernova model successfully reproduces the observed abundance patterns. Moreover, we show that the initial parameters of the progenitor, such as the initial masses and the rotational property, can be constrained by the model, since the stellar yields result from the nucleosynthesis in the outer region of the star, which is significantly affected by the initial parameters. The initial parameter of Pop III stars is of prime importance for the theoretical study of the early universe. Future observation will increase the number of such carbon enhanced iron-deficient stars, and the same analysis on the stars may give valuable information for the Pop III stars that existed in our universe.
\end{abstract}

Keywords. nuclear reactions, nucleosynthesis, abundances - stars: abundances - stars: Population III - stars: rotation

\section{Introduction}

Stars that are firstly formed in the universe are called first stars or Population III (Pop III) stars. Their evolutionary characteristic is important and interesting topic in astrophysics, since high energy photons emitted from the surface initiate the cosmic reionization, and moreover, they are the first nuclear reactor in the universe (for review, Bromm \& Yoshida 2011). Recent development of star formation theory allows us to investigate what is the realistic star formation site of first stars. Hirano et al. (2014) showed that the initial mass range of Pop III stars will be wide from $\sim 10 \mathrm{M}_{\odot}$ to $\sim 1000$ $\mathrm{M}_{\odot}$. Also, Stacy et al. (2013) found that a Pop III star can have a fast rotation speed at its birth.

How can we test the very interesting expectations on Pop III stars, and what are the mass range and the rotational property of Pop III stars that really existed in the universe? A good candidate for the investigation is to use abundance patterns preserved in metalpoor stars. Theoretically, metal-poor stars should be born in a metal-poor, unevolved gas cloud that is expected to show nuclear synthesis features of the metal supplying mother star(s). Therefore, the abundance patterns can be used to constrain theoretical models for nucleosynthesis in the progenitor stars, and the method is called abundance profiling. Such a theoretical study benefits from the large number of observed metal-poor stars. So far, three iron-poor stars that have $[\mathrm{Fe} / \mathrm{H}] \dagger<-5$ are known, they are HE 0107-5240 of $[\mathrm{Fe} / \mathrm{H}]=-5.3$ (Christlieb et al. 2002), HE 1327-2326 of $[\mathrm{Fe} / \mathrm{H}]=-5.7$ (Frebel et al.

$\dagger[\mathrm{A} / \mathrm{B}]=\log _{10}\left(N_{A} / N_{B}\right)-\log _{10}\left(N_{A} / N_{B}\right) \odot, N_{A}$ is a number abundance of a species $\mathrm{A}$. 
2005), and recently found SMSS 0313-6708 of $[\mathrm{Fe} / \mathrm{H}]<-7$ (Keller et al. 2014). Not only the low iron abundance, but also the enhancement of intermediate mass elements, such as carbon, nitrogen, oxygen, sodium, and magnesium, is the characteristic feature of the abundances.

Until now, several works investigated yields from the Pop III stars and the first supernovae (e.g., Umeda \& Nomoto 2005). However, no abundance features has been known that can be used to constrain the initial parameters of Pop III progenitors. Firstly, because of the degeneracy in the explosive yields, the initial mass range of the progenitor has not yet been constrained (Umeda \& Nomoto 2005). Secondly, since no rotating Pop III calculations have been conducted for abundance profiling so far, abundance features showing rotational contribution has not yet obtained. Thus, the aim of this work is to find stellar yields by doing abundance profiling, that are useful to constrain the initial masses and the rotation of the first stars.

\section{Method}

\subsection{Stellar Evolution Calculation}

Evolution of massive zero metallicity stars are calculated by the latest version of the stellar evolution code used in Takahashi et al. (2014). The primordial abundance by Steigman (2007) is used for the initial composition. A wide mass range from $12 \mathrm{M}_{\odot}$ to $140 \mathrm{M}_{\odot}$ is taken in order to cover a likely mass range for all core collapse supernova. Also, stellar rotation is included in the calculation, which affects the yields by effective internal mixing due to several rotational instabilities.

\subsection{Assumptions on Supernova Explosion}

In this work, stellar matter is assumed to be ejected by supernova explosion at the end of the stellar life. First assumption for the ejection is that the only outer part of the star that is weakly bound by gravity is ejected by the explosion. Second assumption is that the explosion is too weak to modify the composition of outer distributed matter by the shock heating. We call a supernova explosion that is responsible for these assumptions as a weak supernova. With these assumptions, stellar yields $M_{i}$ can be calculated by a simple integration using the parameter for the inner boundary $M_{i n}$,

$$
M_{i}\left(M_{i n}\right)=\int_{M_{i n}}^{\mathrm{M}_{\text {surface }}} X_{i}(M) d M,
$$

where $X_{i}$ is a mass fraction distribution of $i$ element. In order to show the depth of the ejection, the parameter $f_{i n}=M_{i n} / M_{\mathrm{CO}}$ is also used in the analysis, here $M_{\mathrm{CO}}$ is a mass of the CO core.

\subsection{Abundance Calculation}

Using the integrated mass $M_{i}$ as the stellar yield, the chemical composition of a second generation star can be calculated as

$$
X_{i, 2 \mathrm{nd}}=\frac{M_{i} / M_{\mathrm{SN}}+X_{i, \mathrm{ISM}} M_{\mathrm{ISM}} / M_{\mathrm{SN}}}{1+M_{\mathrm{ISM}} / M_{\mathrm{SN}}}
$$

where $M_{\mathrm{SN}}=\sum_{i} M_{i}$ and $M_{\mathrm{ISM}}$ are total masses of the ejecta and the ISM, and $D=$ $M_{\mathrm{ISM}} / M_{\mathrm{SN}}$ is a dilution factor. Dilution factors vary with models, and typically become several hundreds for best fitted models for HMP stars. Comparison between calculated abundances and observation is done by using $[\mathrm{i} / \mathrm{j}]$ as the indicator. The solar abundance by Asplund et al. (2009) is used in the comparison. 

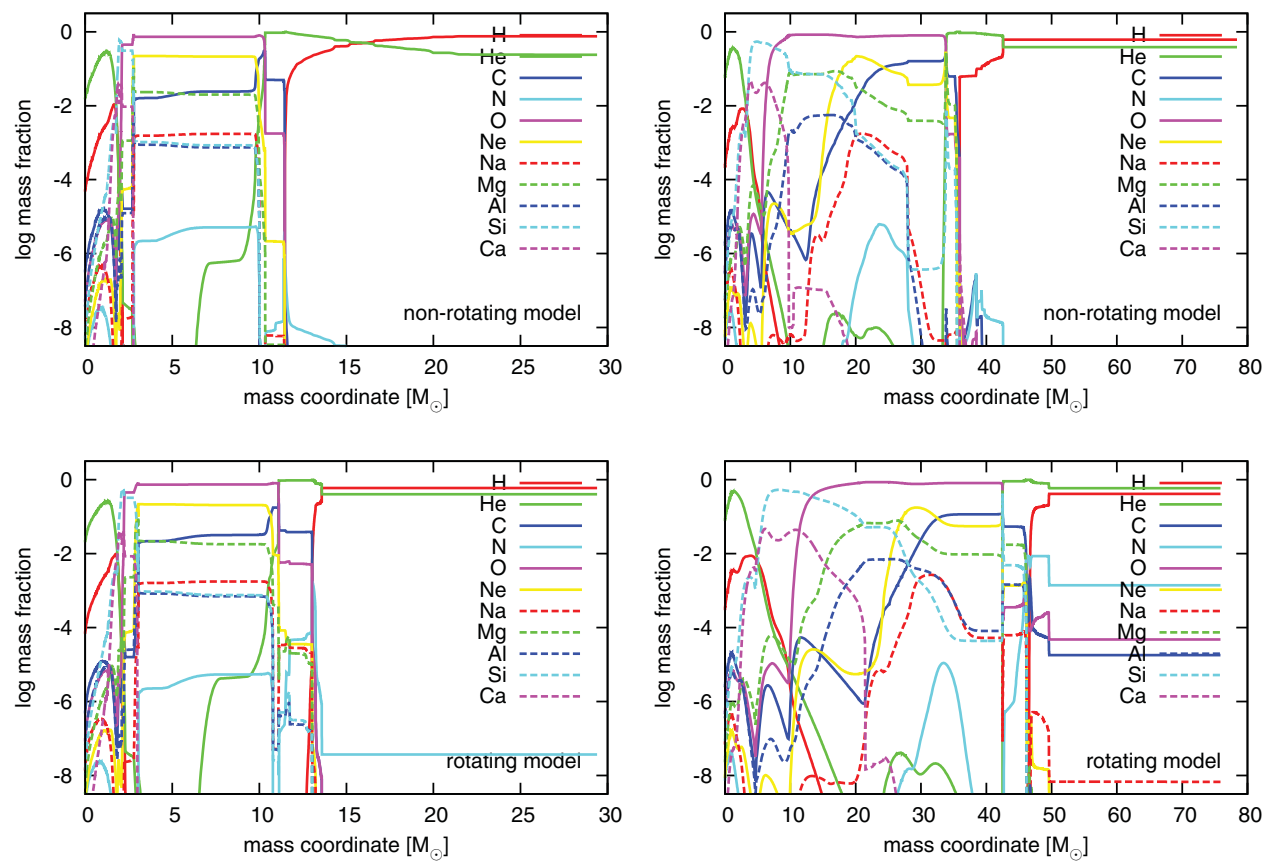

Figure 1. Abundance distributions of rotating and non-rotating, 30 and $80 \mathrm{M}_{\odot}$ models.

\section{Stellar Yields}

Fig 1 shows calculated abundance distribution in models of rotating and non-rotating, 30 and $80 \mathrm{M}_{\odot}$ stars. Important differences are appeared in outer regions of stars, especially in their helium layers.

Carbon and Oxygen All stellar models yield carbon and oxygen in their helium layers. Since abundant helium remains during the evolution in this region, resulting $\mathrm{C} / \mathrm{O}$ ratio becomes large and always exceeds unity. Thus, carbon and oxygen production with a large $[\mathrm{C} / \mathrm{O}]$ can be regarded as a general signature of nucleosynthesis in the outer region of a star.

Magnesium and Silicon In the figure, magnesium and Silicon are produced in $80 \mathrm{M}_{\odot}$ models, and generally, these alpha elements are produced only in massive models. Production and non-production of these alpha elements are, thus, useful to constrain the initial mass of the progenitor.

Sodium and Aluminum Both rotating 30 and $80 \mathrm{M}_{\odot}$ models yield sodium and aluminum in their helium layers, while non-rotators do not. This is due to the rotationally induced mixing in the rotating models. First, carbon and oxygen synthesized by core helium burning are transported into hydrogen burning shell by the rotationally induced mixing. This results in nitrogen production by CNO cycle, and nitrogen distributes in the helium layer. Then alpha capture reactions on nitrogen take place, producing ${ }^{22} \mathrm{Ne}$, a famous neutron source for massive stellar evolution. Finally neutron capture reactions on ${ }^{22} \mathrm{Ne}$ and ${ }^{26} \mathrm{Mg}$ take place, producing ${ }^{23} \mathrm{Na}$ and ${ }^{27} \mathrm{Al}$. Sodium and aluminum enhancement are used to constrain the stellar rotation of the progenitor.

Calcium Only very massive models yield calcium, and the mechanisms are different between rotating and non-rotating progenitors. For rotating models, efficient alpha capture reactions are responsible for the production, and the process is similar to the production of magnesium and silicon. For non-rotating models, efficient proton capture 

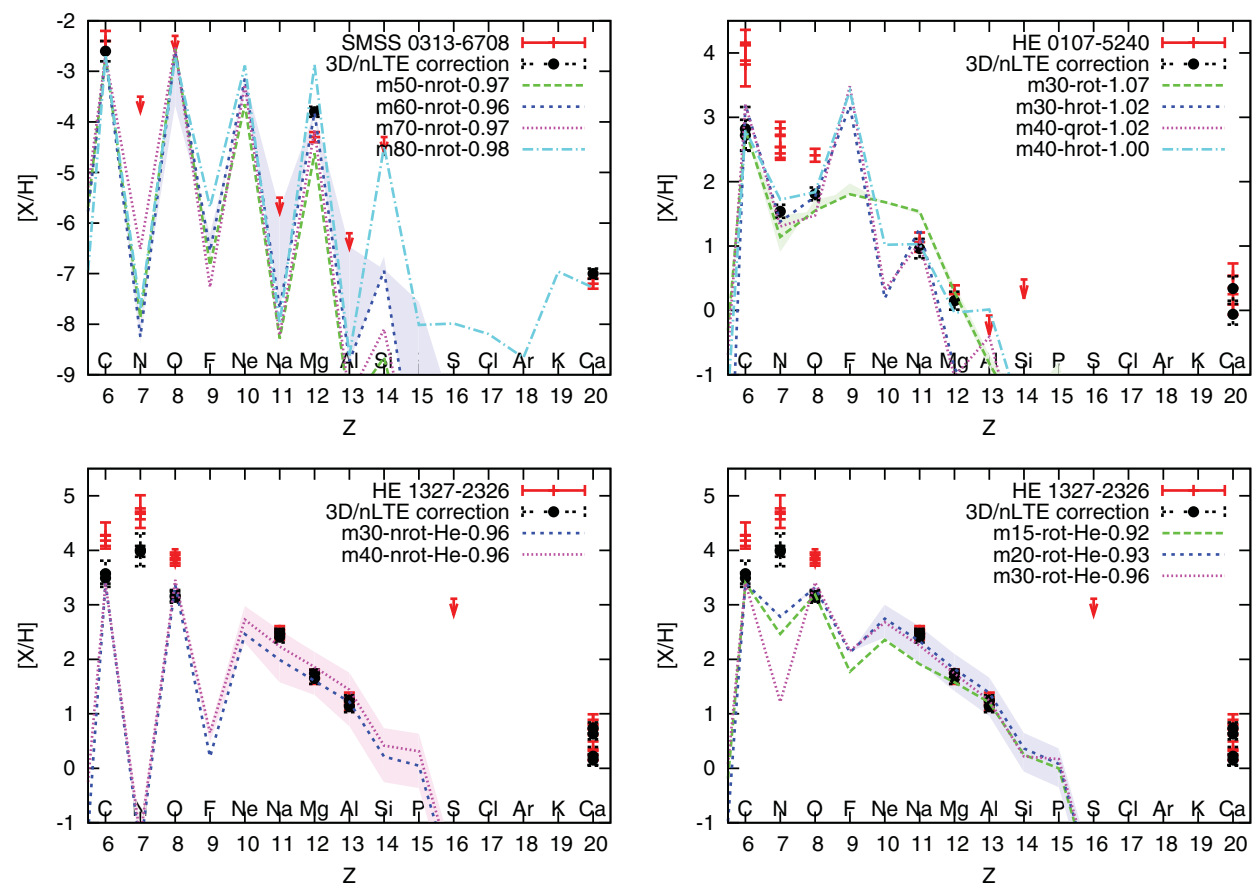

Figure 2. Results of abundance profiling.

reactions take place at the base of the hydrogen burning shell and account for the calcium production.

\section{Abundance Profiling}

SMSS 0313-6708 SMSS 0313-6708, recently found by Keller et al. (2014), is the most iron-deficient star known until now. Iron has not been detected in the star, and very small upper limit of $[\mathrm{Fe} / \mathrm{H}]<-7$ has been reported. Interesting feature of the composition of the star is the enhancement of carbon $([\mathrm{C} / \mathrm{H}] \sim-2.6)$ and magnesium $([\mathrm{Mg} / \mathrm{H}] \sim-3.9)$. In addition, upper limits of $[\mathrm{Na} / \mathrm{Mg}]<-1.2$ and $[\mathrm{Al} / \mathrm{Mg}]<-1.9$ are valuable for the abundance profiling.

First, massive stars of $>100 \mathrm{M}_{\odot}$ stars overproduce magnesium at helium layers. These stars fail to explain the small $[\mathrm{Mg} / \mathrm{C}]$ and thus rejected as the progenitor star. On the other hand, less massive models of $<40 \mathrm{M}_{\odot}$ produce sodium and aluminum besides magnesium, and do not fit with the observation. This is because, magnesium should be ejected from inner carbon burning region in the less massive stars, however, carbon burning simultaneously synthesizes sodium and aluminum, which are not detected in the star. Similarly, the upper limits of sodium and aluminum are used to constrain rotating models. Due to the rotationally induced mixing, all rotating progenitors produce sodium and aluminum in helium layers. Because of the overproduction, no rotating models can explain the observation.

Therefore, a massive, but not too massive, non-rotating star can be a progenitor of SMSS 0313-6708. The non-rotating $60 \mathrm{M}_{\odot}$ gives the best fitting to the abundance pattern from carbon to silicon. Calcium has been detected in the star with a small value of $[\mathrm{Ca} / \mathrm{H}]=-7.0$. If the future observation reveal the enhancement of calcium as well as 
Table 1. Summary of the abundance profiling.

\begin{tabular}{cccccc}
\hline Object & {$[\mathrm{Fe} / \mathrm{H}]$} & $M_{\text {ini }}$ & $f_{\text {in }}$ & Rotation & Dilution Factor \\
\hline SMSS 0313-6708 & $<-7.1$ & $50-80$ & $0.96 \pm 0.04\left(60 \mathrm{M}_{\odot}\right)$ & non-rotating & $1.78 \times 10^{3}-6.09 \times 10^{2}$ \\
& & & $0.98 \pm 0.04\left(80 \mathrm{M}_{\odot}\right)$ & non-rotating & $1.62 \times 10^{3}-1.91 \times 10^{2}$ \\
HE 0107-5240 & -5.3 & $30-40$ & $1.07 \pm 0.06\left(30 \mathrm{M}_{\odot}\right)$ & rotating & $7.84 \times 10^{2}-2.23 \times 10^{2}$ \\
HE 1327-2326 & -5.7 & $20-40$ & $0.96 \pm 0.01\left(40 \mathrm{M}_{\odot}\right)$ & non-rotating & $5.00 \times 10^{2}-4.32 \times 10^{2}$ \\
& & $15-30$ & $0.93 \pm 0.01\left(20 \mathrm{M}_{\odot}\right)$ & rotating & $7.92 \times 10^{2}-7.35 \times 10^{2}$ \\
\hline
\end{tabular}

other intermediate mass elements, then the non-rotating $80 \mathrm{M}_{\odot}$ will be a good candidate for the progenitor, since the model produces calcium by fast proton capture reactions.

HE 1327-2326 and HE 0107-5240 The other two iron-deficient stars, HE 1327$2326([\mathrm{Fe} / \mathrm{H}]=-5.7$, reported by Frebel et al. $(2005))$ and $\mathrm{HE} \mathrm{0107-5240}([\mathrm{Fe} / \mathrm{H}]=-5.3$, reported by Christlieb et al. (2002)), are also compared with the theoretical yields. Plotted data are obtained from Aoki et al. (2006); Frebel et al. (2006, 2008); Bonifacio et al. (2012) for HE1327-2326, and from Christlieb et al. (2004); Bessell et al. (2004); Bessell \& Christlieb (2005), and for 3D collection from Collet et al. (2006).

Abundance pattern from sodium to aluminum is useful to constraint the progenitor of HE 1327-2326. The pattern, and the relative abundance with carbon (e.g., $[\mathrm{Mg} / \mathrm{C}]=$ -2.7) can be well represented by ejecting tiny fraction of material in the carbon burning region of $\sim 15-40 \mathrm{M}_{\odot}$ models. Thus, rotating $15-30 \mathrm{M}_{\odot}$ and non-rotating $20-40 \mathrm{M}_{\odot}$ models give good fits for HE 1327-2326. HE 0107-5240 has a highly enhanced carbon abundance relative to oxygen, $[\mathrm{O} / \mathrm{C}]=-1.4$. Such a pattern can only be explained by a mass ejection from a very outer region of the progenitor star. In addition, sodium abundance is much higher than magnesium abundance in the star, $[\mathrm{Na} / \mathrm{Mg}]=0.8$, and stellar rotation can be responsible for the enhancement. In our models, the rotating $30 \mathrm{M}_{\odot}$ star with $f_{\text {in }}=$ 1.01-1.13 gives the best fit to the star.

\section{References}

Aoki, W., Frebel, A., Christlieb, N., et al. 2006, ApJ 639, 897

Asplund, M., Grevesse, N., Sauval, A. J., \& Scott, P. 2009, ARA\& $A$ 47, 481

Bessell, M. S. \& Christlieb, N. 2005, in V. Hill, P. Francois, \& F. Primas (eds.), From Lithium to Uranium: Elemental Tracers of Early Cosmic Evolution, Vol. 228 of IAU Symposium, pp $237-238$

Bessell, M. S., Christlieb, N., \& Gustafsson, B. 2004, ApJ (Letters) 612, L61

Bonifacio, P., Caffau, E., Venn, K. A., \& Lambert, D. L. 2012, A\&A 544, A102

Bromm, V. \& Yoshida, N. 2011, ARA\& A 49, 373

Christlieb, N., Bessell, M. S., Beers, T. C., et al. 2002, Nature 419, 904

Christlieb, N., Gustafsson, B., Korn, A. J., et al. 2004, ApJ 603, 708

Collet, R., Asplund, M., \& Trampedach, R. 2006, ApJ (Letters) 644, L121

Frebel, A., Aoki, W., Christlieb, N., et al. 2005, Nature 434, 871

Frebel, A., Christlieb, N., Norris, J. E., Aoki, W., \& Asplund, M. 2006, ApJ (Letters) 638, L17

Frebel, A., Collet, R., Eriksson, K., Christlieb, N., \& Aoki, W. 2008, ApJ 684, 588

Hirano, S., Hosokawa, T., Yoshida, N., et al. 2014, ApJ 781, 60

Keller, S. C., Bessell, M. S., Frebel, A., et al. 2014, Nature 506, 463

Stacy, A., Greif, T. H., Klessen, R. S., Bromm, V., \& Loeb, A. 2013, MNRAS 431, 1470

Steigman, G. 2007, Annual Review of Nuclear and Particle Science 57, 463

Takahashi, K., Umeda, H., \& Yoshida, T. 2014, ArXiv e-prints

Takahashi, K., Yoshida, T., \& Umeda, H. 2013, ApJ 771, 28

Umeda, H. \& Nomoto, K. 2005, ApJ 619, 427 


\section{Discussion}

LANGER: Would your $50-80 M_{\odot}$ stars not form black holes rather than supernovae?

TAKAHASHI: Still proper predictions of fates of massive stars are difficult. We just assume in this work that the outer matter of the first stars is ejected somehow. The mechanism may be a weakly energetic supernova, a jet-like explosion, or a failed supernova.

LANGER: How complete is your parameters space, e.g., what about binaries or mixing during the supernova explosion?

TAKAhASHI: Currently, binarity is too complicated to implement in our work, though it could have important effects on the results.

MoRAVVEJI: $Z=0$ stars must have masses around $0.8 M_{\odot}$, not $80 M_{\odot}$, otherwise they wouldn't have stayed until the present days, so that we observe them. Then, how can you compare yields of $80 M_{\odot}$ models with a $\sim 0.8 M_{\odot}$ star?

TAKAHASHI: These stars are not essentially first generation, but from next generations. So, they already have some enrichments from previous generations.

MoravVEJI: To model second generation of metal-free stars, do you start from the predicted yields of the first generation stars?

TAKAHASHI: No, we do not do that here.

Meynet: Do you have predictions about the ${ }^{12} \mathrm{C} /{ }^{13} \mathrm{C}$ ratio? This is a very constraining quantity for comparisons with observations.

TAKAHASHI: I will check the data. My expectation is that because the weak supernova yields have moderate amount of ${ }^{12} \mathrm{C}$ from the layers in addition to ${ }^{13} \mathrm{C}$ from $\mathrm{CNO}$ regions, ${ }^{13} \mathrm{C} /{ }^{12} \mathrm{C}$ will show a slight enhancement.

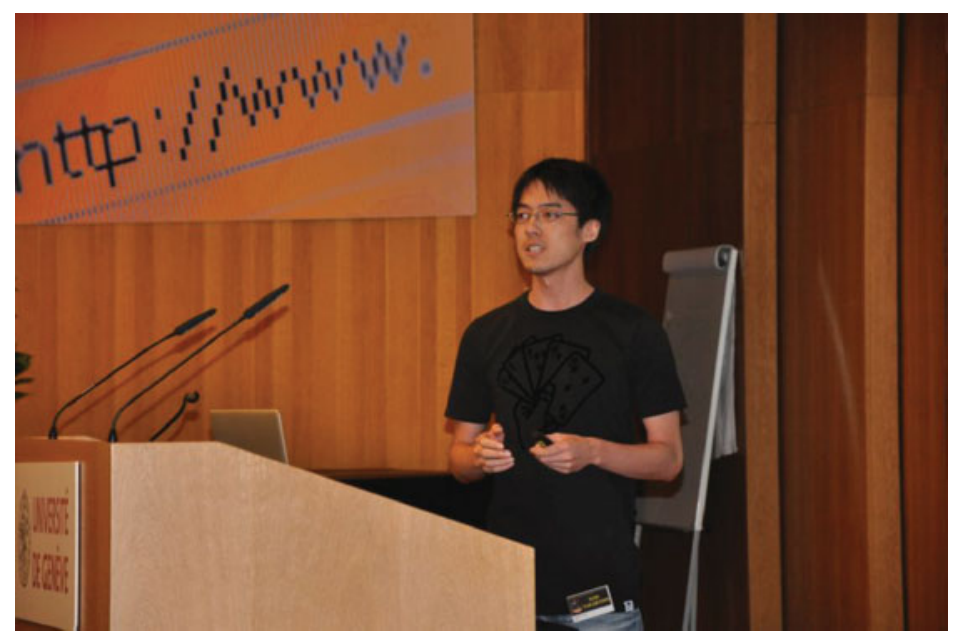

Koh Takahashi 